\title{
ALTERNATIVA ANALÍTICA A LA DETERMINACIÓN DE TENSIONES BAJO ZAPATAS RECTANGULARES CON FLEXIÓN BIAXIL Y DESPEGUE PARCIAL
}

\author{
(EXPLICIT STRESSES UNDER RECTANGULAR DETACHED FOOTINGS WITH \\ BIAXIAL BENDING)
}

Ramón Irles Más, Profesor titular de universidad Dt. de Inga de la Construcción, O.P. e Infraestructura Urbana

Francisco Irles Más, Profesor asociado

Dt. de Expresión Gráfica y Cartografía

UNIVERSIDAD DE ALICANTE. ESPANA

\section{RESUMEN}

Como alternativa a las técnicas numéricas para la obtención de la distribución de presiones bajo una zapata rectangular con flexocompresión biaxil y presencia de despegues, supuesta lineal, en este artículo se han obtenido fórmulas explícitas, excepto para el caso de despegue en un solo vértice, en el cual el problema se reduce al inmediato de resolver una ecuación polinómica de grado 8 y una sola variable, con garantía y unicidad de solución en el intervalo de definición del problema; caso que, a su vez, presenta situaciones particulares explicitables.

\section{SUMMARY}

The calculation of stresses under rectangular footings with biaxial bending is carried out by solving the 3-equations system that sets up the load and soil stresses equilibrium. When a planar soil stresses distribution is assumed, and the area is in full compression, the above system is linear and its solution inmediate. With increasing eccentricities, some corners of the footing can get detached from the soil, the system becoming nonlinear, and difficulties arise in its resolution. Classic answers to this problem have been found through the numerical (iterative) solution of the system, or by getting nondimensional graphs for direct calculation, whose sketches also require a numerical solution.

In the paper an analytical alternative is presented which will lead to obtaining explicit solutions for these stresses and neutral axis location.

\section{1.-INTRODUCCIÓN. ESTADO DE LA CUESTIÓN}

El dimensionamiento en planta de las zapatas suele realizarse admitiendo una distribución plana para las tensiones transmitidas al terreno. Esta hipótesis resulta bastante verosímil en terrenos rocosos o arcillosos y arenosos altamente consolidados, y no tanto para estos últimos cuando están normalmente consolidados; en tal caso, una distribución parabólica de tensiones puede resultar más próxima a la realidad.

Admitida, no obstante, la hipótesis lineal, el cálculo de tensiones para una zapata y cargas dadas es directo e inmediato en los casos en que no existe despegue del terreno (es decir, cuando toda la base de la zapata comprime el terreno, con más o menos intensidad), ya que entonces las tensiones pueden calcularse fácil- 
mente a partir del sistema de ecuaciones lineales que exige el equilibrio entre las acciones sobre la base de la zapata y la reacción del terreno (Fig. 1):

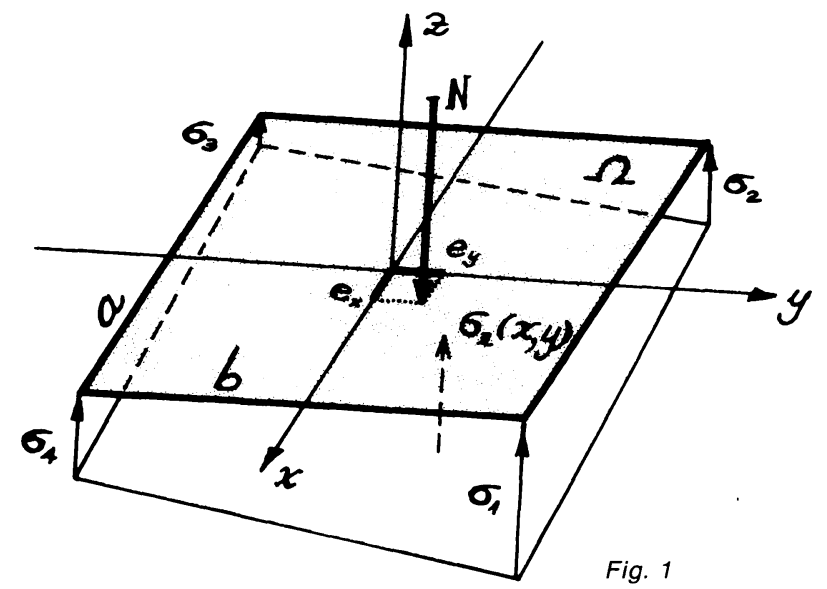

$\iint_{\Omega} \sigma_{z}(x, y) d x d y=N$

$\iint_{\Omega} x \sigma=(x, y) d x d y=M_{y}=N e^{[1]}$ $\iint_{\Omega} y \sigma=(x, y) d x d y=M x=N e y$

el cual conduce a la conocida fórmula:

$$
\sigma_{z}(x, y)=\frac{N}{A}+\frac{N e_{x}}{I_{y}} x+\frac{N_{y}}{I_{x}} y
$$

donde: $A=a b$

$$
\begin{aligned}
& I_{x}=a b^{3 / 12} \\
& I_{y}=b a^{3} / 12
\end{aligned}
$$

Esta fórmula queda sin validez en el caso de que se presente despegue parcial de alguna zona de la base, lo cual es asumido cuando [2] indica tracciones. Ello ocurre cuando la resultante, N, se sitúa fuera del Núcleo Central, zona bien conocida y delimitada (Fig. 2):

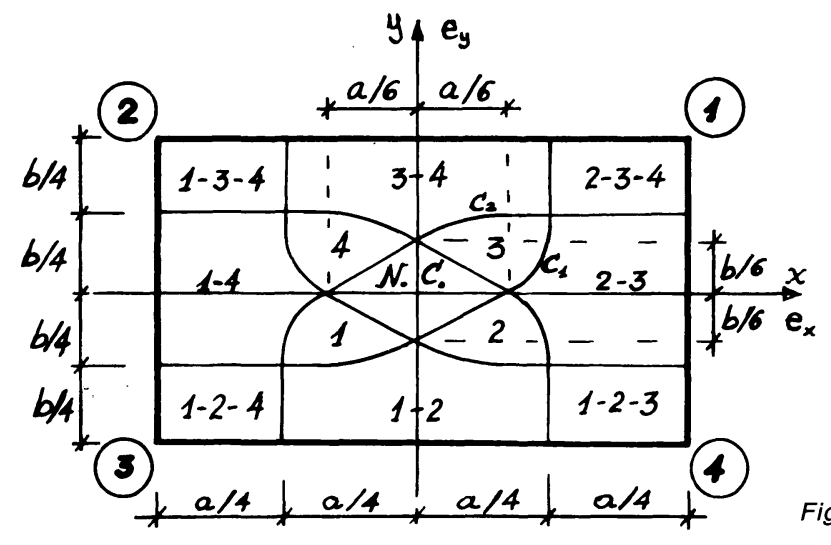

$$
\left|\frac{e_{x}}{a}\right|+\left|\frac{e_{y}}{b}\right| \leq \frac{1}{6}
$$

y tiene como consecuencia la no linealidad del sistema (1).

Hace tiempo que se conoce (1), la zonificación completa de la planta de la zapata, generalización del concepto de núcleo central, que permite clasificar el tipo de despegue. Ésta se reproduce en la Fig. 2, con indicación de los vértices despegados que corresponden a cada zona de paso de la resultante; sus fronteras son rectas de ecuación inmediata y curvas que pueden definirse en forma paramétrica, como veremos. 
Para el caso particular de zapatas rectangulares, con flexocompresión monoaxil y presencia de despegues, el cálculo es también directo e inmediato a partir de dicho sistema (1), que ya no es lineal, pero se resuelve fácilmente (Fig. 3):

$$
\begin{aligned}
& N=\frac{1}{2} \sigma_{1} d b \quad \sigma_{1}=\frac{2 N}{d b}=\frac{2 N}{3 b\left(\frac{M_{y}}{N}-\frac{a}{2}\right)} \\
& M_{\gamma}-N \frac{a}{2}=\frac{1}{2} \sigma_{1} d b \frac{d}{3}=N \frac{d}{3} \quad d=3\left(\frac{M_{y}}{N}-\frac{a}{2}\right)
\end{aligned}
$$

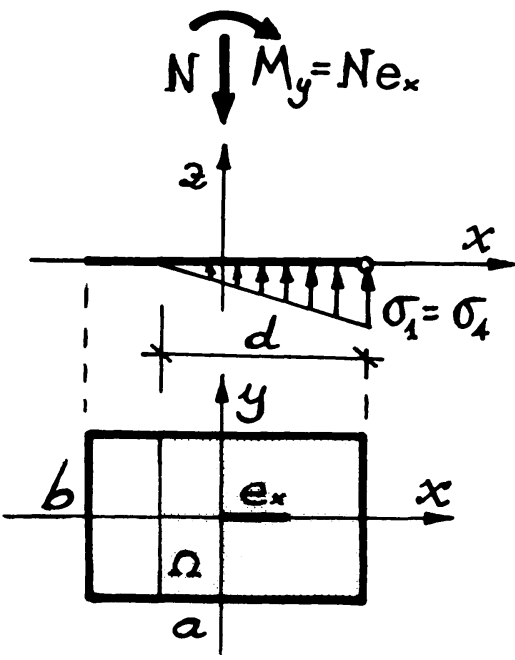

Finalmente, para los casos de flexión biaxil con presencia de despegue, la solución del citado sistema no lineal [1] se complica, habiendo sido abordada su resolución por las siguientes vías:

1. Mediante un cálculo iterativo en función de dos parámetros independientes que identifiquen la línea límite de despegue, (2), (7) y otros; obsérvese que la incógnita restante, por ejemplo la presión máxima, puede eliminarse siempre dividiendo miembro a miembro las ecuaciones del sistema [1].

2. Pueden obtenerse varias soluciones "directas", (6) y (9) (incluso para el caso de distribución parabólica de presiones (10) ), a base de soluciones numéricas (iterativas) del sistema [1] para valores discretos de determinados parámetros geométricos del problema, que permiten dibujar ciertas figuras para determinar gráficamente, y con relativa precisión, la posición de la fibra neutra y la presión máxima.

Puede encontrarse bibliografía en castellano al respecto en las referencias (2), (3), (4), (5) y (8).

En este artículo se desarrolla una alternativa analítica a dichos procedimientos, que conduce a soluciones explícitas, salvo en un único caso, que queda reducido a una ecuación polinómica de grado 8 y una sola variable, con garantía y unicidad de solución en el intervalo de definición del problema.

\section{2.-OBTENCIÓN EXPLÍCITA DE LA DISTRIBUCIÓN DE PRESIONES}

Se estudian a continuación los casos posibles de despegue en 3, 2 ó 1 vértices de la zapata, obteniendo en cada caso, para geometría y carga dadas, la posición de la fibra neutra y presiones transmitidas. Dada la doble simetria del problema, se realiza este desarrollo para el primer cuadrante de la zapata.

\section{1.-Despegue en tres vértices}

Este es el caso más sencillo y se llega con facilidad a fórmulas explícitas para el valor de tensión máxima y los que definen la zona de despegue (Fig. 4). 


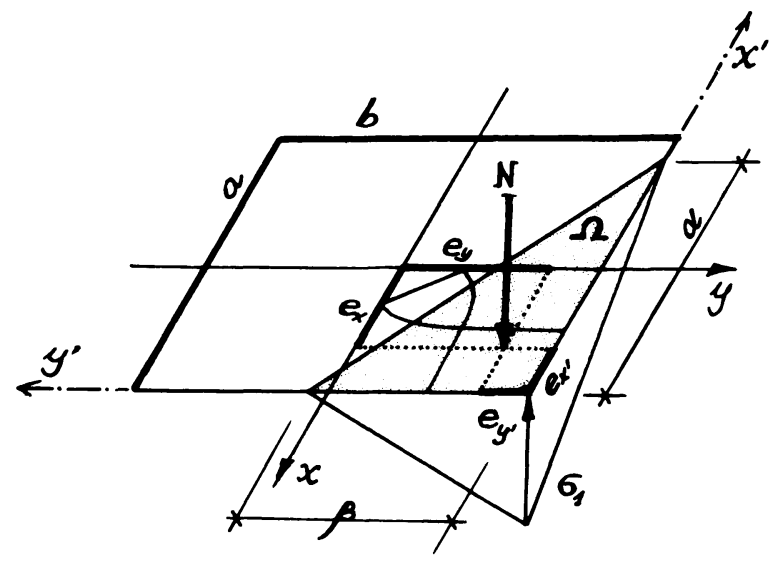

Fig. 4

Con la nomenclatura indicada, las ecuaciones [1] toman la forma:

$$
\begin{aligned}
& \mathrm{N}=\frac{\alpha \beta \sigma_{1}}{6} \\
& M_{x}=\frac{\alpha \beta^{2} \sigma_{1}}{24} \\
& M_{y}^{\prime}=\frac{\alpha^{2} \beta \sigma_{1}}{24}
\end{aligned}
$$

sistema no lineal que puede resolverse así:

$$
\begin{aligned}
& e_{x}=\frac{M_{y}{ }^{\prime}}{N}=\frac{\alpha}{4} \\
& e_{x}=\frac{a}{2}-\frac{\alpha}{4} \\
& \alpha=2 a-4 e_{x} \\
& e_{y}{ }^{\prime}=\frac{M_{x}{ }^{\prime}}{N}=\frac{\beta}{4} \\
& e_{y}=\frac{b}{2}-\frac{\beta}{4} \\
& \beta=2 b-4 e_{y}
\end{aligned}
$$

y de la primera ecuación:

$$
\sigma_{1}=\frac{6 N}{\alpha \beta}=\frac{3 N}{2\left(a-2 e_{x}\right)\left(b-2 e_{y}\right)}
$$




\section{2.-Despegue en dos vértices}

\section{a) Despegue en 2-3}

Este caso, más complicado que el anterior, también permite obtener fórmulas explícitas. Operando por diferencia de tetraedros semejantes, con la nomenclatura de la Fig. 5 , tenemos las siguientes ecuaciones de equilibrio [1]:

$$
\begin{aligned}
& N=\frac{1}{6} \alpha \beta \sigma_{1}\left[1-\frac{(\beta-b)^{3}}{\beta^{3}}\right] \\
& M_{x}^{\prime}=\frac{\alpha \beta \sigma_{1}}{24}\left[\beta-\frac{(\beta-b)^{3}(\beta+3 b)}{\beta^{3}}\right] \\
& M_{y}^{\prime}=\frac{\alpha \beta \sigma_{1}}{24}\left[\alpha-\frac{\alpha(\beta-b)^{4}}{\beta^{4}}\right]
\end{aligned}
$$

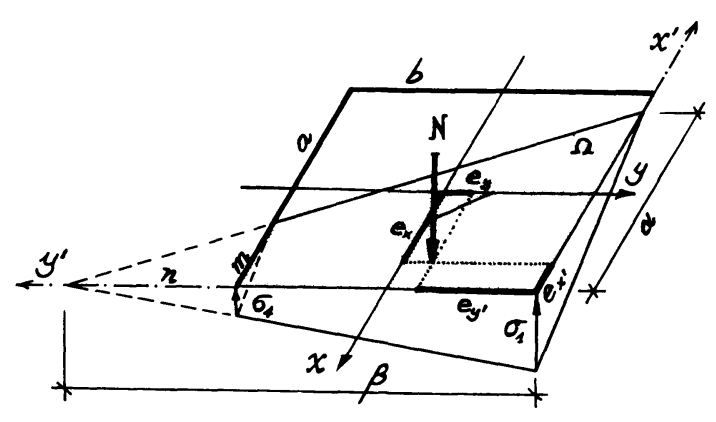

Fig. 5

Dividiendo la segunda ecuación por la primera, se obtiene otra que sólo depende de $\beta$ :

$$
e_{y},=\frac{M x^{\prime}}{N}=\frac{1}{4} \frac{3 b^{3}-8 \beta b^{2}+6 \beta^{2} b}{3 \beta^{2}-3 \beta b+b^{2}}
$$

llamando

$$
\begin{aligned}
& \mu=\frac{4 e_{y}}{b}=2-\frac{4 e_{y}}{b} \\
& \delta=\beta / b>1
\end{aligned}
$$

y tras algunas manipulaciones algebraicas, se llega a la ecuación de segundo grado:

$$
\begin{gathered}
(6-3 \mu) \delta^{2}-(8-3 \mu) \delta+3-\mu=0 \\
\delta=\frac{8-3 \mu+\sqrt{12 \mu-3 \mu^{2}-8}}{12-6 \mu}
\end{gathered}
$$

de la cual:

(el signo se decide a partir de casos particulares de fácil cálculo). Quedando así explícito: 
Dividiendo la tercera por la primera, se puede explicitar ahora $\alpha$ en función de $\delta$ :

$$
e_{x}^{\prime}=\frac{M_{y}}{N}=\frac{\alpha}{4} \frac{\beta^{4}-(\beta-b)^{4}}{\beta^{4}-\beta(\beta-b)^{3}}=\frac{\alpha}{4 \delta} \frac{\delta^{4}-(\delta-1)^{4}}{\delta^{3}-(\delta-1)^{3}}
$$

de donde, habida cuenta de que $e_{x}^{\prime}=\frac{a}{2}-e_{x}$ :

$$
\alpha=\frac{4 \delta\left[\delta^{3}-(\delta-1)^{3}\right]}{\delta^{4}-(\delta-1)^{4}}\left(\frac{a}{2}-e_{x}\right)
$$

también explícito, en función de $e_{x}, e_{y}$.

Finalmente, de la primera ecuación, por ejemplo, se obtiene:

$$
\sigma_{1}=\frac{6 \mathrm{~N} \delta^{2}}{\alpha \mathrm{b}\left[\delta^{3}-(\delta-1)^{3}\right]}
$$

y de las relaciones de semejanza:

$$
\begin{aligned}
& \sigma_{4}=\sigma_{1}(1-1 / \delta) \\
& \mathrm{m}=\alpha(1-1 / \delta) \\
& \mathrm{n}=\mathrm{b}(\delta-1)
\end{aligned}
$$

\section{b) Despegue en 3-4}

Mediante un desarrollo totalmente paralelo, con la nomenclatura de la Fig. 6 se obtiene:

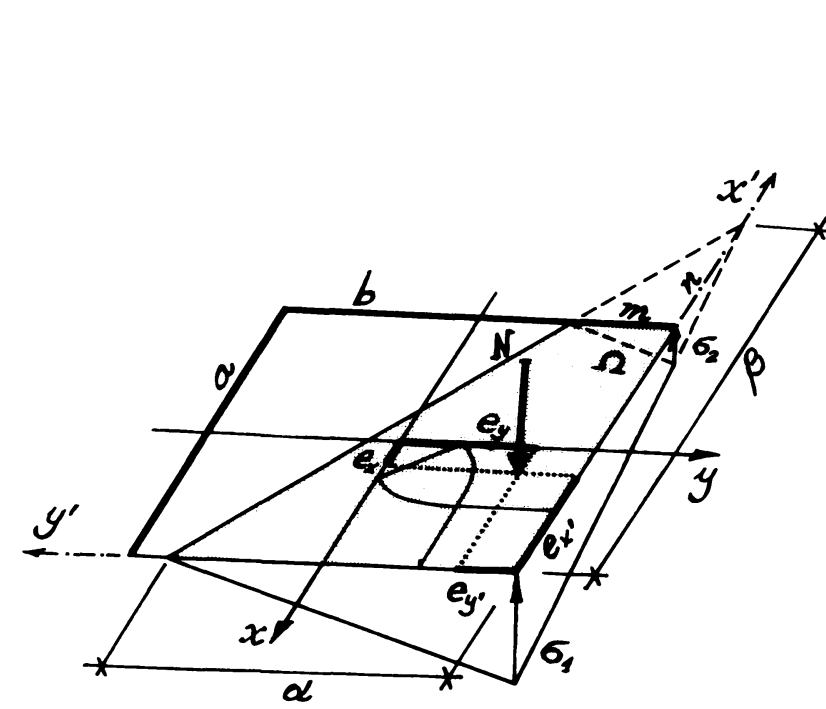

$$
\begin{aligned}
& \mu=2-4 e \times / a \\
& \delta=\left[8-3 \mu+\sqrt{12 \mu-3 \mu^{2}-8}\right] /(12-6 \mu)
\end{aligned}
$$$$
\beta=a \delta
$$$$
\alpha=\frac{4 \delta\left[\delta^{3}-(\delta-1)^{3}\right]}{\delta^{4}-(\delta-1)^{4}}\left(\mathrm{~b} / 2-\mathrm{e}_{\mathrm{y}}\right)
$$$$
\sigma_{1}=\frac{6 N \delta^{2}}{\alpha a\left[\delta^{3}-(\delta-1)^{3}\right]}
$$$$
\sigma_{2}=\sigma_{1}(1-1 / \delta)
$$$$
\mathrm{m}=\alpha(1-1 / \delta)
$$

Fig. 6

$n=a(\delta-1)$ 


\section{3-Despegue de un solo vértice (3)}

Este es el caso más complejo. Con la nomenclatura de la Fig. 7 tendremos:

plano de tensiones:

$\sigma_{z}^{\prime}\left(x^{\prime}, y^{\prime}\right)=-\sigma_{3}\left(1-x^{\prime} / a \alpha-y^{\prime} / b \beta\right)[3]$

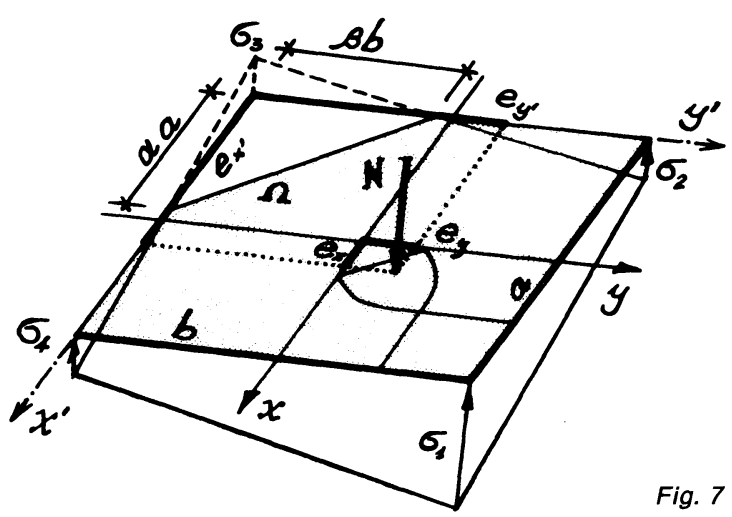

ecuaciones de equilibrio [1]:

$$
\begin{aligned}
& N=\frac{a b \sigma_{3}}{2 \alpha \beta}\left[\alpha+\beta-2 \alpha \beta+\alpha^{2} \beta^{2} / 3\right] \\
& M_{x}=\frac{a b^{2} \sigma_{3}}{24 \alpha \beta}\left[6 \beta-12 \alpha \beta+8 \alpha+\alpha^{2} \beta^{3}\right] \\
& M_{y}{ }^{\prime}=\frac{a^{2} b \sigma_{3}}{24 \alpha \beta}\left[6 \alpha-12 \alpha \beta+8 \beta+\beta^{2} \alpha^{3}\right]
\end{aligned}
$$

y puestas en forma de excentricidades, o sea, dividiendo miembro a miembro las segunda y tercera ecuaciones por la primera, con lo que elimina la incógnita $\sigma_{3}$, y en los ejes principales:

$$
\begin{aligned}
& e_{x}{ }^{\prime}=M_{y} \cdot / N \\
& e_{y}=M_{x} \cdot / N \\
& e_{x}=e_{x} \cdot-a / 2=\frac{a \beta}{4} \frac{2-2 \alpha^{2} \beta+\beta \alpha^{3}}{3 \alpha+3 \beta-6 \alpha \beta+\alpha^{2} \beta^{2}} \\
& e_{y}=e_{y} \cdot-b / 2=\frac{b \alpha}{4} \frac{2-2 \alpha \beta^{2}+\alpha \beta^{3}}{3 \alpha+3 \beta-6 \alpha \beta+\alpha^{2} \beta^{2}}
\end{aligned}
$$

(*) De estas ecuaciones pueden obtenerse las paramétricas de las fronteras curvas en la zonificación de la zapata, particularizando $\beta=1$ ó $\alpha=1$, respectivamente:

$$
C_{1} \equiv\left\{\begin{array}{l}
e_{x}=\frac{a}{4} \frac{2-2 \alpha^{2}+\alpha^{3}}{\alpha^{2}-3 \alpha+3} \\
e_{y}=\frac{b}{4} \frac{2 \alpha-\alpha^{2}}{\alpha^{2}-3 \alpha+3} \\
\alpha \in] 0,1[
\end{array}\right.
$$

$$
C_{2} \equiv\left\{\begin{array}{lll}
e_{x} & =\frac{a}{4} & \frac{2 \beta-\beta^{2}}{\beta^{2}-3 \beta+3} \\
e_{y}= & \frac{b}{4} & \frac{2-2 \beta^{2}+\beta^{3}}{\beta^{2}-3 \beta+3} \\
\beta \in] & 0,1[
\end{array}\right.
$$

http://informesdelaconstruccion.revistas.csic.es 
Quedando el siguiente sistema no lineal de dos ecuaciones con dos incógnitas:

$$
\begin{aligned}
& \left(3 \alpha+3 \beta-6 \alpha \beta+\alpha^{2} \beta^{2}\right) 4 e_{x} / a=2 \beta-2 \alpha^{2} \beta^{2}+\beta^{2} \alpha^{3} \\
& \left(3 \alpha+3 \beta-6 \alpha \beta+\alpha^{2} \beta^{2}\right) 4 e_{y} / b=2 \alpha-2 \alpha^{2} \beta^{2}+\alpha^{2} \beta^{3}
\end{aligned}
$$

Hasta el momento no se ha encontrado solución general explícita para el mismo, pero con algunas transformaciones algebraicas el problema se simplifica notablemente. En efecto, considerando el sistema equivalente constituido por las ecuaciones suma y cociente, miembro a miembro, de las [5]:

$$
\begin{aligned}
& A\left(3 \alpha+3 \beta-6 \alpha \beta+\alpha^{2} \beta^{2}\right)=2(\alpha+\beta)-4 \alpha^{2} \beta^{2}+(\alpha+\beta) \alpha^{2} \beta^{2} \\
& C\left(2 \alpha-2 \alpha^{2} \beta^{2}+\alpha^{2} \beta^{3}\right)=2 \beta-2 \alpha^{2} \beta^{2}+\beta^{2} \alpha^{3}
\end{aligned}
$$

donde:

$$
\begin{aligned}
& \left.A=4\left(e_{x} / a+e_{y} / b\right) \in\right] 2 / 3,2[ \\
& \left.C=b e_{x} / a e_{y} \in\right] 0, \infty[
\end{aligned}
$$

y efectuando el cambio de variables:

$$
\begin{array}{lll}
\mathrm{u}=\alpha+\beta & (0<\mathrm{u}<2) & {[7 . a]} \\
\mathrm{v}=\alpha \beta & (0<\mathrm{v}<1) & {[7 . b]}
\end{array}
$$

la [6.a] queda:

$$
A\left(3 u-6 v+v^{2}\right)=2 u-4 v^{2}+u v^{2}
$$

de la cual se puede explicitar:

$$
u=u(v)=v \frac{6 A-(A+4) v}{3 A-2-v^{2}}
$$

Por otra parte, sustituyendo $\alpha=\mathrm{u}-\beta$ en la [6.b]:

$$
C\left(2 u-2 \beta-2 v^{2}+\beta v^{2}\right)=2 \beta-2 v^{2}+u v^{2}-\beta v^{2}
$$

de donde se puede explicitar:

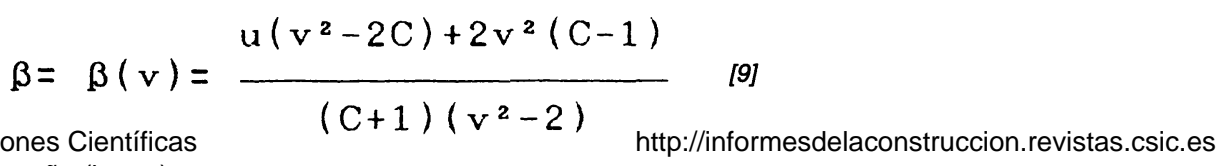


$y$, por tanto:

$$
\alpha=\alpha(\mathrm{v})=\mathrm{u}(\mathrm{v})-\beta(\mathrm{v})=\frac{\mathrm{u}\left(\mathrm{Cv^{2 }}-2\right)-2 \mathrm{v}^{2}(\mathrm{C}-1)}{(\mathrm{C}+1)\left(\mathrm{v}^{2}-2\right)}
$$

Finalmente, para la resolución del sistema queda por satisfacer (7.b):

$$
\alpha(v) \beta(v)=v
$$

ecuación en una sola variable, que, después de racionalizar, resulta ser polinómica de grado 9 en v:

$$
P_{9}(v)=0
$$

En ella, v $=0$ es solución, independientemente del valor de $\mathrm{A}$ y $\mathrm{C}$, como resulta inmediato comprobar de [8], [9], [10] y [11], y corresponde a $\alpha=0$ ó $\beta=0$, es decir, a resultantes pasando por la frontera del núcleo central, caso ya conocido. Para otros valores $v \neq 0$, eliminando el factor común $v$ de [12] queda:

$$
P_{B}(v)=\sum_{1=0}^{8} A_{1} v^{1}=0
$$

con:

$$
\begin{aligned}
& A_{0}=4(C+1)^{2}(3 A-2)^{2} \\
& A_{1}=-144 A^{2} C \\
& A_{2}=12 A\left[2 C(5 A+6)+\left(C^{2}+1\right)(6-9 A)\right] \\
& A_{3}=-4 C(A+4)^{2}+72 A^{2}\left(C^{2}+1\right)+8(C-1)^{2}\left(6 A^{2}-A-2\right) \\
& A_{4}=-12 A(C-1)^{2}(3 A-4)+(C+1)^{2}\left(9 A^{2}+12 A-8\right)-24 A\left(C^{2}+1\right)(A+4) \\
& A_{5}=-36 A^{2} C+2\left(C^{2}+1\right)(A+4)^{2}-2(C-1)^{2}\left(8+4 A-3 A^{2}\right) \\
& A_{0}=6 A\left[2 C(A+1)+C^{2}+1\right] \\
& A_{7}=-C(A+4)^{2}-2(A+2)(C-1)^{2} \\
& A_{8}=(C+1)^{2}
\end{aligned}
$$

polinomio de grado 8, para el cual puede comprobarse (mediante exhaustiva verificación numérico-gráfica, dada la complejidad algebraica del problema), que (Fig. 8): 


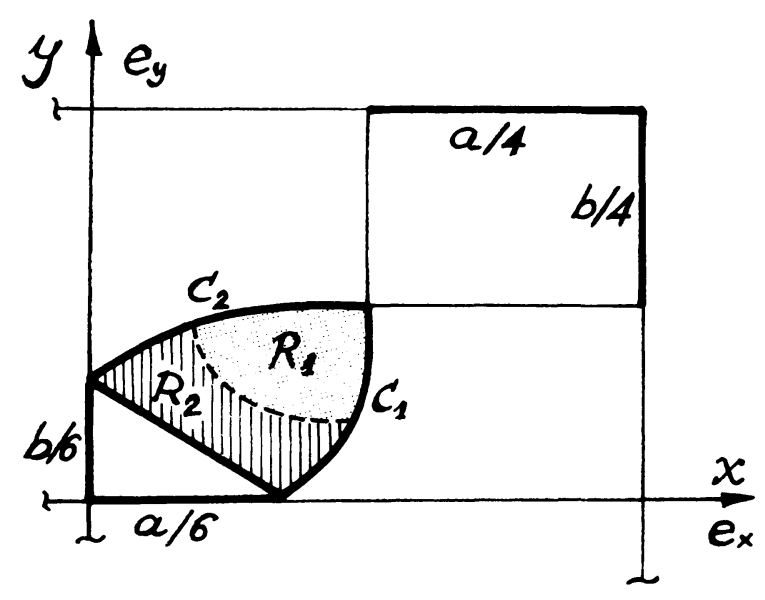

Fig. 8

a) En un recinto $R_{1}$ de la zona correspondiente:

$$
\begin{aligned}
& \mathrm{P}_{8}(0)>0 \quad \forall\left(e_{x}, e_{y}\right) \in R_{1} \\
& \mathrm{P}_{8}(1)<0 \quad \forall\left(e_{x}, e_{y}\right) \in R_{1} \\
& \left.\frac{\mathrm{dP}_{8}(\mathrm{v})}{\mathrm{dv}}<0 \quad \forall\left(e_{x}, e_{y}\right) \in R_{1} \quad y \quad \forall v \in\right] 0,1[
\end{aligned}
$$

existiendo, por tanto, garantía y unicidad de solución en $\mathrm{R}_{1}$.

b) En el resto de la zona, $R_{2}: P_{8}$ (v) presenta dos, y sólo dos, cambios de signo en $\left.v \epsilon\right] 0,1$ [, $\bar{V}$ (ex, ey) $\epsilon R_{2}$ existiendo, por tanto dos, y sólo dos, soluciones $0<v_{1}<v_{2}<1$; pero la solución mayor, v2, es extraña al proble$\mathrm{ma}$, pues conduce a valores de $\mathrm{u}_{2}<0$, fuera del rango del problema [7.a].

Resultando, por tanto, inmediata, aunque no explícita, en general, hoy por hoy, la solución de [13], y, teniendo en cuenta [8], [9] y [10], la del sistema [5]. Ahora puede obtenerse de cualquiera de las [4], por ejemplo de [4.a], el valor ficticio:

$$
\sigma_{3}=\frac{2 N \alpha \beta}{a b\left[\alpha+\beta-2 \alpha \beta+\alpha^{2} \beta^{2} / 3\right]}
$$

y del plano de tensiones [3]:

$$
\begin{aligned}
& \sigma_{1}=\sigma_{3}(1 / \alpha+1 / \beta-1) \\
& \sigma_{2}=\sigma_{3}(1 / \beta-1) \\
& \sigma_{4}=\sigma_{3}(1 / \alpha-1)
\end{aligned}
$$

Únicamente para el caso particular de excentricidades relativas iguales,

$$
\frac{e_{x}}{a}=\frac{e_{y}}{b} \quad\left(C=1, A=8 e_{x} / a\right)
$$


el grado de polinomio se reduce a 4:

$$
P_{4}(v)=\sum_{1=0}^{4} B_{1} \quad v^{1}=0
$$

con:

$$
\begin{array}{ll}
B_{0}=(3 A-2)^{2} & B_{3}=-(A / 2+2)^{2} \\
B_{1}=-9 A^{2} & B_{4}=1 \\
B_{2}=3 A^{2}+6 A+4 &
\end{array}
$$

y resulta explicitable su solución, obteniendo sucesivamente:

$$
\begin{aligned}
& p=-3 B_{3}{ }^{2} / 8+B_{2} \\
& q=B_{3} 3 / 8-B_{3} B_{2} / 2+B_{1} \\
& r=-3 B_{3}{ }^{4} / 256+B_{3}{ }^{2} B_{2} / 16-B_{3} B_{1} / 4+B_{0} \\
& p^{\prime}=\left(p^{2}-4 r\right) / 16-p^{2} / 12 \\
& q^{\prime}=p^{3} / 108-\left(p^{2}-4 r\right) p / 96-q^{2} / 64 \\
& \varnothing=\sqrt{-\left(p^{1} / 3\right)^{3}} \\
& \theta=\operatorname{ar} \cos \left(-q^{\prime} / 2 \varnothing\right)
\end{aligned}
$$

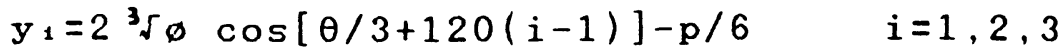

$y$, finalmente:

$$
\begin{aligned}
& \mathrm{v}_{1}=S\left(-\sqrt{\mathrm{y}_{1}}+\sqrt{\mathrm{y}_{2}}+\sqrt{\mathrm{y}_{3}}\right)-\mathrm{B}_{3} / 4 \\
& \mathrm{v}_{2}=S\left(\sqrt{\mathrm{y}_{1}}+\sqrt{\mathrm{y}_{2}}-\sqrt{\mathrm{y}_{3}}\right)-\mathrm{B}_{3} / 4 \\
& \mathrm{v}_{3}=S\left(-\sqrt{\mathrm{y}_{1}}-\sqrt{\mathrm{y}_{2}}-\sqrt{\mathrm{y}_{3}}\right)-\mathrm{B}_{3} / 4 \\
& \mathrm{v}_{4}=S\left(\sqrt{\mathrm{y}_{1}}-\sqrt{\mathrm{y}_{2}}+\sqrt{\mathrm{y}_{3}}\right)-\mathrm{B}_{3} / 4
\end{aligned}
$$$$
\operatorname{con} S=q /|q|
$$

debiendo recordar la discusión de soluciones antes comentada $(\mathrm{V} \epsilon] 0,1[, \mathrm{u} \epsilon] 0,2[$ ) para seleccionar la raíz solución del problema. 


\section{3.-EJEMPLOS}

A continuación se presentan dos ejemplos correspondientes al caso más complejo de despegue en un solo vértice.

3.1.ex/a $=0.2$ ey/b=0.1

Los coeficientes de (13) son:

$$
\begin{array}{rlrl}
\mathrm{A}=1.2 & \mathrm{C}=2 \\
\mathrm{~A}_{0}=92.16 & \mathrm{~A}_{1}=-414.72 & \mathrm{~A}_{2}=\mathrm{A}_{3}=345.6 & \mathrm{~A}_{4}=-568.8 \\
\mathrm{~A}_{5}=149.76 & \mathrm{~A}_{8}=99.36 & \mathrm{~A}_{7}=-60.48 & \mathrm{~A}_{8}=9
\end{array}
$$

obteniéndose como solución $v=0.3252$ y, por tanto, de [8], [9] y [10]:

$$
u=1.1991 \quad \alpha=0.4146 \quad \beta=0.7845
$$

y las tensiones son:

$$
\sigma_{1}=2.9933 \mathrm{~N} / \mathrm{ab} \quad \sigma_{2}=0.3061 \mathrm{~N} / \mathrm{ab} \quad \sigma_{4}=1.5731 \mathrm{~N} / \mathrm{ab}
$$

quedando sin compresión el vértice 3, con los despegues dados por $\alpha$ a y $\beta$ b.

\section{2. $-e x / a=e y / b=0.2$}

En este caso particular se obtiene $A=1.6 \quad C=1$.

Los coeficientes del polinomio $\mathrm{P}_{4}(\mathrm{~V})$ son:

$$
\mathrm{B}_{0}=7.84 \quad \mathrm{~B}_{1}=-23.04 \quad \mathrm{~B}_{2}=21.28 \quad \mathrm{~B}_{3}=-7.84 \quad \mathrm{~B}_{4}=1
$$

y sucesivamente:

$$
\begin{array}{lllll}
\mathrm{p}=-1.7696 & \mathrm{q}=0.1413 & \mathrm{r}=0.1572 & \mathrm{p}^{\prime}=-0.1045 & \mathrm{q}^{\prime}=-0.005487 \\
\emptyset=0.006504 & \theta=65.050 & \mathrm{y}_{1}=0.6418 & \mathrm{y}_{2}=0.002018 & \mathrm{y}_{3}=0.2409 \\
\mathrm{v}_{1}>1 & \mathrm{v}_{2}>1 & \mathrm{v}_{3}=0.6231 & \mathrm{v}_{4}>1 &
\end{array}
$$

quedando explícita la solución válida v3 $\epsilon$ ] 0,1[. A partir de ella, igual que antes:

$$
u=1.5787 \quad \alpha=\beta=0.7894 \quad \sigma_{1}=4.1373 \mathrm{~N} / \mathrm{ab} \quad \sigma_{2}=\sigma_{4}=0.7199 \mathrm{~N} / \mathrm{ab}
$$




\section{4.-BIBLIOGRAFÍA}

(1). "Foundation design".

W.C. Teng

Prentice-Hall, Inc., Englewood Cliffs. New Jersey, 1962.

(2). "La losa rígida sobre base elástica".

F. Morán

Ficha E-37 del Servicio de Cálculo. Informes de la Construcción, 183, pp. 131 y 132. Agosto 1966.

(3). "Determinación de las presiones a que está sometido un terreno, bajo una zapata rectangular solicitada por una flexión biaxial".

F. Sebastián

Cuadernos de estructuras, 2.a serie, Tomo V n. 1-2. 1967.

(4). "Presiones ejercidas sobre el terreno por una zapata rectangular sometida a compresión excéntrica". F. Baratech

Cuadernos de estructuras, 2. serie, Tomo VI n. 9-10. 1970.

(5). "Hormigón Armado"

P.J. Montoya, A.G. Meseguer y F. Morán

Gustavo Gili, Barcelona. Ed. 7 a 13, 1973 a 1991.

(6). "Bearing pressures on bridge footings".

M. Hackman

Civil Engineering. Nov. 1977.

(7). "Ingeniería de cimentaciones".

R.B. Peck, W.E. Hanson y T.H. Thornburn

Limusa. México, 1982.

(8). "Problemas que origina el diseño y comportamiento de zapatas de medianería y esquina".

E. Abdilla

Tesis doctoral. Universidad Politécnica de Valencia, 1983.

(9). "Pressure under eccentrically loaded footings".

B.O. Hardin

Civil Engineering for Practicing and Design Engineers.

Vol. 3, pp. 511-516. 1984.

(10). "Rectangular foundations subjected to biaxial bending".

D.M.A. Vitone and A.J. Valsangkar

Civil Engineering for Practicing and Design Engineers.

Vol. 5, pp. 799-814. 1986. 